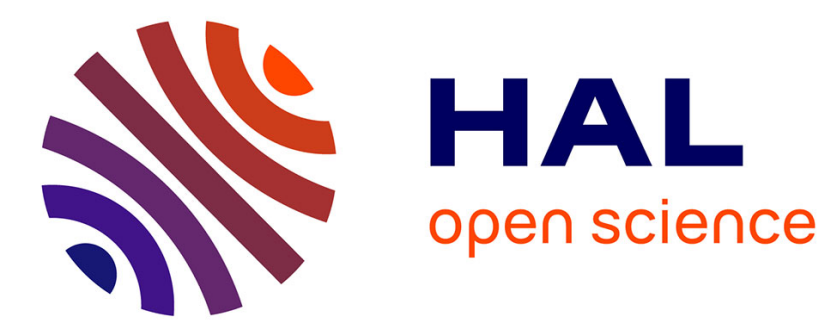

\title{
Blind Source Separation in Polarimetric SAR Interferometry
}

\author{
Gabriel Vasile, Leandro Pralon
}

\section{To cite this version:}

Gabriel Vasile, Leandro Pralon. Blind Source Separation in Polarimetric SAR Interferometry. IGARSS 2016 - IEEE International Geoscience and Remote Sensing Symposium, Jul 2016, Beijing, China. pp.4. hal-01387500

\section{HAL Id: hal-01387500 https://hal.science/hal-01387500}

Submitted on 25 Oct 2016

HAL is a multi-disciplinary open access archive for the deposit and dissemination of scientific research documents, whether they are published or not. The documents may come from teaching and research institutions in France or abroad, or from public or private research centers.
L'archive ouverte pluridisciplinaire HAL, est destinée au dépôt et à la diffusion de documents scientifiques de niveau recherche, publiés ou non, émanant des établissements d'enseignement et de recherche français ou étrangers, des laboratoires publics ou privés. 


\title{
BLIND SOURCE SEPARATION IN POLARIMETRIC SAR INTERFEROMETRY
}

\author{
Gabriel Vasile and Leandro Pralon \\ Grenoble-Image-sPeach-Signal-Automatics Lab, GIPSA-lab \\ CNRS / Grenoble-INP, Grenoble, France \\ gabriel.vasile@gipsa-lab.grenoble-inp.fr
}

\begin{abstract}
Polarimetric incoherent target decomposition aims in accessing physical parameters of illuminated scatters through the analysis of target coherence or covariance matrix. In this framework, Independent Component Analysis (ICA) was recently proposed as an alternative method to Eigenvector decomposition to better interpret non-Gaussian heterogeneous clutter (inherent to high resolution SAR systems). Until now, the two main drawbacks reported of the aforementioned method are the greater number of samples required for an unbiased estimation, when compared to classical Eigenvector decomposition and the inability to be employed in scenarios under Gaussian clutter assumption. First, a Monte Carlo approach is performed in order to investigate the bias in estimating the Touzi Target Scattering Vector Model (TSVM) parameters when ICA is employed. A RAMSES X-band image acquired over Brétigny, France is taken into consideration to investigate the bias estimation under different scenarios. Finally, some results in terms of POLinSAR coherence optimization [1] in the context of ICA are proposed.
\end{abstract}

Index Terms - Polarimetry, SAR, Interferometry, ICA

\section{INTRODUCTION}

Polarimetric target decomposition is one of the most powerful and widespread tools for POLSAR image interpretation. The analysis of the interaction between the illuminated area and the transmitted waveform, to each polarimetric state of the latter, allows for a better prediction of the basic scattering mechanisms present on the scene, and to more efficiently propose classification, detection and geophysical parameter inversion algorithms.

Many methods have been proposed in the literature to both decompose an image pixel into basic target vectors as well as to correctly retrieve quantitative information from them (parametrization). Concerning the latter, Cloude and Pottiers parameters (entropy, alpha and anisotropy) [2] and Touzi Target Scattering Vector Model [3] are the most employed ones, whose usefulness have already been demon-

Thanks to the French Aerospace Lab (ONERA) for providing the Brétigny POLinSAR data. strated by several authors. Regarding the decomposition, the algorithms are mainly classified in either coherent, if they are based on the scattering matrix analysis, or incoherent, if their interest lies in the Hermitian, semidefinite positive coherence or covariance matrix. Incoherent target decomposition (ICTD) theory assumes that the scattering process in most natural media is a combination of coherent speckle noise and random vector scattering effects. Therefore, not only a statistical analysis is often required, but also it is a common practice to associate to the imaging cell the concept of average or dominant scattering mechanisms [4]. The Eigenvector based ICTD manages to decompose an image pixel into the three most dominant scatters from the averaged coherence matrix. Furthermore, it has an intrinsic property that the derived scatters are orthogonal and uncorrelated, which for Gaussian clutters also means independence. The drawback of this kind of method emerge when the clutter is not Gaussian [5,6] or not composed by orthogonal mechanisms, situations where the performance of the algorithm could be compromised.

\section{BLIND SOURCE SEPARATION STRATEGY IN POLSAR}

In [7], a new Blind Source Separation strategy to polarimetric target decomposition was presented by incorporating the Independent Component Analysis (ICA). The ICA is a blind source separation technique based on higher order statistical moments and cumulants whose utility has already been explored in many different research areas, such as wireless communications, feature extraction and brain imaging applications [8]. The results presented in [7] proved it to be a very promising area in polarimetry, mainly when non-Gaussian heterogeneous clutters (inherent to high resolution SAR systems) are under study. The theoretical potential in estimating similar entropy and first component, when compared to traditional eigenvector decomposition, but rather a second most dominant component independent with respect to the first one and unconstrained by the orthogonality introduces an alternative way of physically interpreting a polarimetric SAR image.

The referred method is briefly summarized in three main steps: data selection, based on the statistical classification of 
the POLSAR image; estimation of independent components and parametrization of the derived target vectors.

As stated in [7], the major drawback of the proposed method is the size of the observation dataset, which has to be somewhat larger than the size of the sliding window used in the well established methods. This constraint led the authors in [7] to use an unsupervised classification algorithm rather than relying on a very large sliding window, jeopardizing the effectiveness of the method.

The use of a classification algorithm limits the performance of the method in the sense that the image is segmented in a priori defined number of classes with variable sizes, what can compromise the estimation of the target vectors parameters and, as a consequence, the correct interpretation of the scatters present in the area under study. One of the implications of the employment of a classification algorithm is that a class can contain more samples than it needs for a correct estimation of targets parameters, meaning that spatial resolution, highly degraded with the use of this approach, is worse than it could be. On the other hand, if a class do not contain samples enough, the parameters estimated can be biased, meaning that the values derived do not comply with ground truth.

Within this context, this paper considers a Monte Carlo simulation approach to evaluate the optimal size of a sliding window for various medias, simple ones composed by basic scatters such as helix, dipole, dihedral and trihedral and more complex ones like Surface, Double Bounce and Volume returns. The simulation procedure is similar to the one presented in [9] to evaluate the bias of multilook effect on Cloude and Pottier [2] parameters in Eigenvector based polarimetric SAR decomposition. An unsupervised classification algorithm is employed to identify within a RAMSES Xband image, sets of samples characterizing Surface, Double Bounce and Volume type of average scattering mechanisms. The mixing matrix and the covariance matrix for each of the aforementioned complex type of scatters are estimated using proper algorithms (Non-Circular Complex Fast-ICA algorithm and Fixed-point algorithm, respectively) and used to bootstrap random samples for the Monte Carlo simulation approach. It is important to highlight that, as reported in [7], the mixing matrix and the covariance matrix are potentially different, thus the set of random samples used in the ICA and Eigenvector decomposition analysis are not the same.

\section{BLIND SOURCE SEPARATION STRATEGY IN POLINSAR}

In case of POLinSAR data, the same ICA algorithm as proposed in [7] is emplyed, except that the dimension of the BSS problem is increased by two. The ICA techniques use a set of observation vectors $(\mathbf{x})$ to retrieve the sources vector $(\mathbf{s})$ and the mixing matrix (A), which gives the share of the sources in the observed process:

$$
\left[\begin{array}{l}
x_{1}^{M} \\
x_{2}^{M} \\
x_{3}^{M} \\
x_{1}^{S} \\
x_{2}^{S} \\
x_{3}^{S}
\end{array}\right]=\left[\begin{array}{llllll}
A_{11} & A_{12} & A_{13} & A_{14} & A_{15} & A_{16} \\
A_{21} & A_{22} & A_{23} & A_{24} & A_{25} & A_{26} \\
A_{31} & A_{32} & A_{33} & A_{34} & A_{35} & A_{36} \\
A_{41} & A_{42} & A_{43} & A_{44} & A_{45} & A_{46} \\
A_{51} & A_{52} & A_{53} & A_{54} & A_{55} & A_{56} \\
A_{61} & A_{62} & A_{63} & A_{64} & A_{65} & A_{66}
\end{array}\right] \cdot\left[\begin{array}{c}
s_{1}^{M} \\
s_{2}^{M} \\
s_{3}^{M} \\
s_{1}^{S} \\
s_{2}^{S} \\
s_{3}^{S}
\end{array}\right]
$$

If we introduce the spatially averaged coherency matrix of the POLinSAR observation vector as $\mathbf{T}_{6}$, the mixing matrix A can be represented as the factorization of the covariance matrix:

$$
\mathbf{T}_{6}=\mathbf{A A}^{\dagger}=\left[\begin{array}{ll}
\mathbf{T}_{11} & \Omega_{12} \\
\Omega_{12}^{* T} & \mathbf{T}_{22}
\end{array}\right]
$$

with $\mathbf{T}_{11}$ being the master POLSAR coherency matrix, $\mathbf{T}_{22}$ the slave POLSAR coherency matrix and $\Omega_{12}$ the interferometric coherency matrix.

After computing these three matrices from the previously derived mixing matrix, it is now possible to directly apply the POLinSAR coherence optimization proposed in [1].

\section{POLSAR EXPERIMENTAL RESULTS}

An unsupervised classification algorithm developed for highly textured POLSAR data [10] is employed to identify within a RAMSES X-band image acquired over Brétigny, France, sets of samples characterizing Surface, Double Bounce and Volume type of average scattering mechanisms. Fig. 1 presents the referred area in Red $(\mathrm{HH}+\mathrm{VV})$, Green $(\mathrm{HV})$ and Blue (HH-VV) and the classification algorithm output.

Analyzing the $\mathrm{H} / \alpha$ feature space, it is possible to concluded that Class 1 is mainly composed by Volume scatters, Class 5 is mainly composed by Surface and Class 6 is mainly composed by Double-Bounce scatters. Therefore samples, corresponding to each class were extracted from the referred set and the mixing matrix and covariance matrix were estimated for each of the described classes $(c=1,2,3)$ of mechanisms.

The parameters of the distribution are then extracted and they are used to generate simulated texture vectors to bootstrap random samples for the Monte Carlo simulation approach. The remaining steps of the simulated data generation are the same as previously described. It is important to highlight that, as reported in [7], the mixing matrix and the covariance matrix are potentially different, thus the set of random samples used in the ICA and Eigenvector decomposition analysis are not the same.

Fig. 2 presents the results of the polarimetric decomposition using both ICA and Eigenvector decomposition (PCA) as a function of the number of looks. 


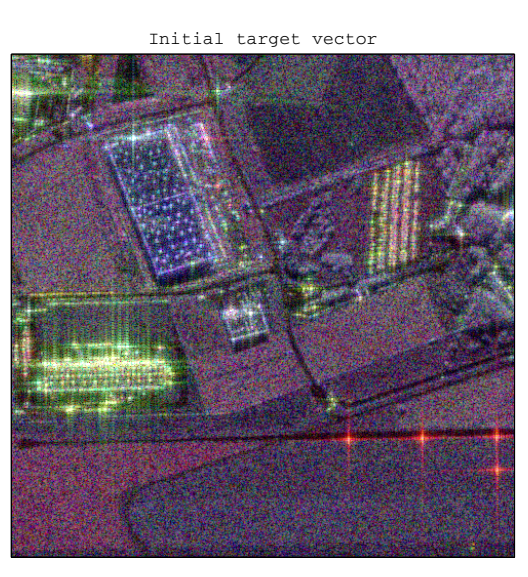

(a)

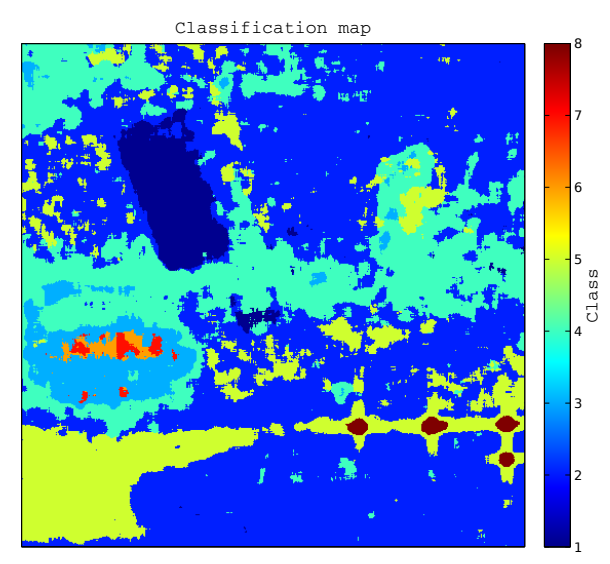

(b)

Fig. 1. Brétigny area under study: (a) RGB image, Red (HH+VV), Green (HV), Blue (HH-VV); (b) Statistical classification algorithm output.

\section{POLINSAR EXPERIMENTAL RESULTS}

Figs. 3 and 4 illustrate the coherence optimization results obtained using the proposed BSS technique - local sliding neighborhood approach, both in terms of optimized coherence and the associated interferometric phases. On can notice the unwrapped phase is converted to DEM using the ambiguity height (around $200 \mathrm{~m}$ for this POLinSAR data set). The obtained results are in good agreement with the available ground truth (top of the building at about $10 \mathrm{~m}$ ).

For example, the hight difference between the 2nd and the 1st component shows that the two scattering mechanisms (building edge and ground) are well separated in Fig. 4-(b). Similar behavior has been reported in [11].

For quantitative performance assessment, the normalized log-ratio [12] between the ICA and PCA derived optimized
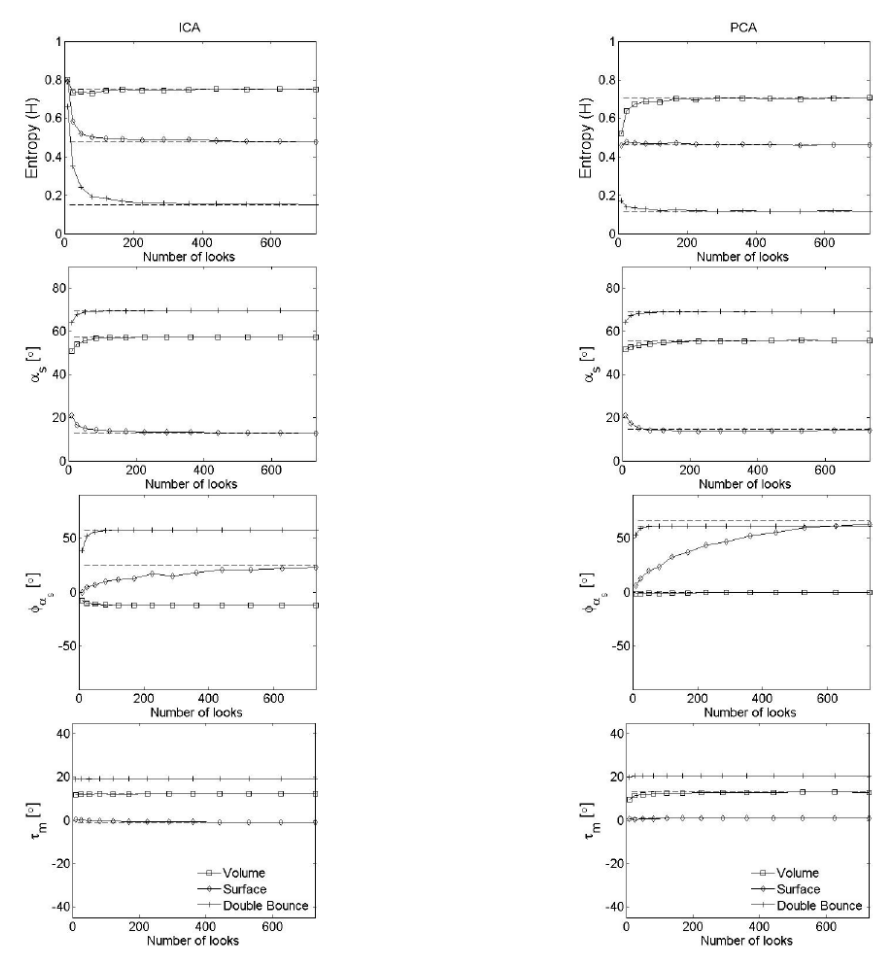

Fig. 2. Entropy and Touzi TSVM parameters derived with ICA and Eigenvector polarimetric target decomposition (PCA) for a complex clutter types: Surface, Double-Bounce and Volume.

coherences is computed as:

$$
R=\sum_{(i, j) \in \mathbf{I}} 20 \log \left(\frac{1}{3} \sum_{k=1 . .3} \frac{c_{k}^{o p t_{I C A}}(i, j)}{c_{k}^{o p t_{P C A}}(i, j)}\right)
$$

The ICA revels an improvement in $R$ of exactly $27.05 \mathrm{~dB}$ computed over the entire test POLinSAR image $(500 \times 500$ pixels).

\section{CONCLUSION}

This paper presented a new framework for applying BSS techniques with POLinSAR data for polarimetric coherence optimization and associated interferometric phases estimation.

After analyzing the POLSAR estimation bias function of the number of looks, the POLinSAR mixing matrix is derived using the Non-Circular Complex Fast-ICA algorithm and Fixed-point algorithm.

The obtained results show improvements in terms of the derived optimized coherences and, in the same time, they remain consistent with the actual ground truth. 


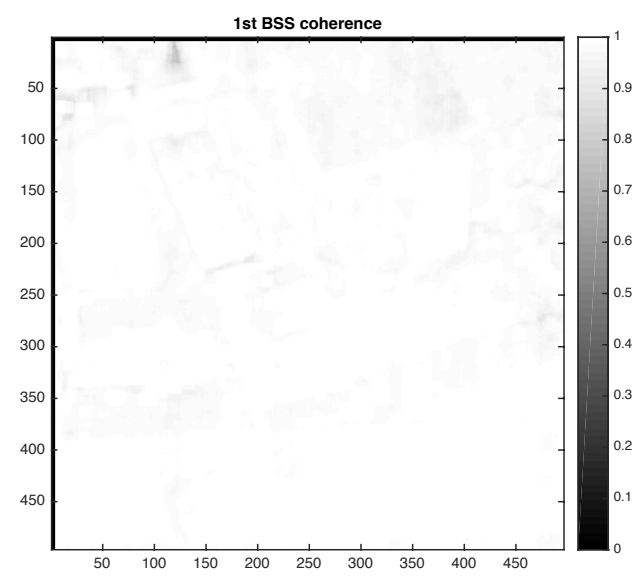

Fig. 3. Brétigny area under study: ICA optimized coherence - 1st component.

\section{REFERENCES}

[1] S. R. Cloude and K. P. Papathanassiou, "Polarimetric SAR interferometry," IEEE Transactions on Geoscience and Remote Sensing, vol. 36, no. 5, pp. 1551-1565, 1998.

[2] S. R. Cloude and E. Pottier, "An entropy based classification scheme for land applications of polarimetric SAR," IEEE Transactions on Geoscience and Remote Sensing, vol. 35, no. 1, pp. 68-78, 1997.

[3] R. Touzi, "Target scattering decomposition in terms of rollinvariant target parameters," IEEE Transactions on Geoscience and Remote Sensing, vol. 45, no. 1, pp. 73-84, 2007.

[4] S. R. Cloude and E. Pottier, "A review of target decomposition theorems in radar polarimetry," IEEE Transactions on Geoscience and Remote Sensing, vol. 32, no. 6, pp. 498-518, 1996.

[5] G. Vasile, J.-P. Ovarlez, F. Pascal, C. Tison, L. Bombrun, M. Gay, and E. Trouvé, "Normalized coherency matrix estimation under the SIRV model. Alpine glacier POLSAR data analysis," in Proceedings of the IEEE International Geoscience and Remote Sensing Symposium, Boston, USA, 2008, vol. 1, pp. I74-I77.

[6] G. Vasile, F. Pascal, J.-P. Ovarlez, P. Formont, and M. Gay, "Optimal parameter estimation in heterogeneous clutter for high resolution polarimetric SAR data," IEEE Geoscience and Remote Sensing Letters, vol. 8, no. 6, pp. 1046-1050, 2011.

[7] N. Besic, G. Vasile, J. Chanussot, and S. Stankovic, "Polarimetric incoherent target decomposition by means of independent component analysis," IEEE Transactions on Geoscience and Remote Sensing, vol. 53, no. 3, pp. 1236-1247, 2015.

[8] A. Hyvarinen, J. Karhunen, and E. Oja, Independent Component Analysis, John Wiley and Sons, 2001.

[9] J. S. Lee, L. Ainsworth, J. P. Kelly, and C. Lopez-Martinez, "Evaluation and bias removal of multilook effect on Entropy/Alpha/Anisotropy in polarimtric SAR decomposition,"

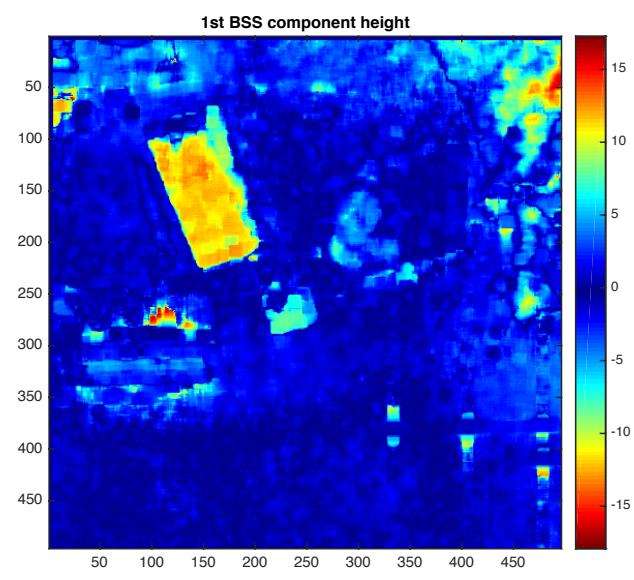

(a)

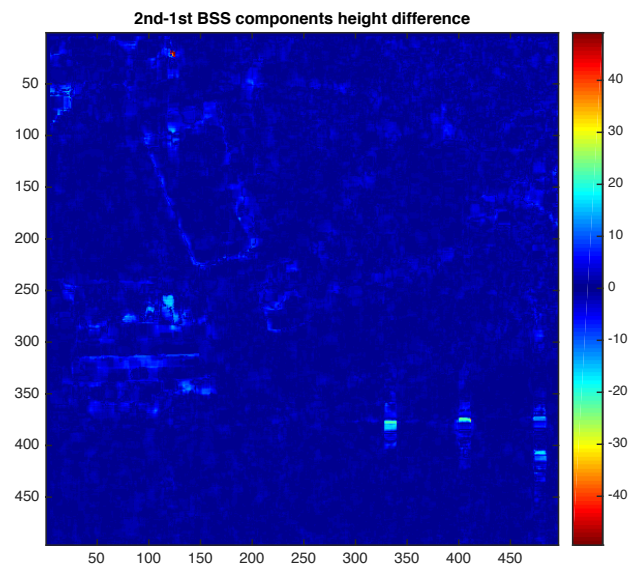

(b)

Fig. 4. Brétigny area under study: Digital Elevation Model (DEM) after ICA coherence optimization - (a) 1st component, (b) height difference between the 2 nd and the 1 st component.

IEEE Transactions on Geoscience and Remote Sensing, vol. 46, no. 10, pp. 3039-3052, 2008.

[10] P. Formont, F. Pascal, G. Vasile, J.-P. Ovarlez, and L. FerroFamil, "Statistical classification for heterogeneous polarimetric SAR images," IEEE Journal of Selected Topics in Signal Processing, vol. 5, no. 3, pp. 398-407, 2011.

[11] E. Colin, C. Titin-Schnaider, and W. Tabbara, "An interferometric coherence optimization method in radar polarimetry for high-resolution imagery," IEEE Transactions on Geoscience and Remote Sensing, vol. 44, no. 1, pp. 167-175, 2006.

[12] R. Touzi, A. Lopes, and P. Bousquet, "A statistical and geometrical edge detector for SAR images," IEEE Transactions on Geoscience and Remote Sensing, vol. 26, no. 6, pp. 764$773,1988$. 